\title{
Polarisation and propagation of lion roars in the dusk side magnetosheath
}

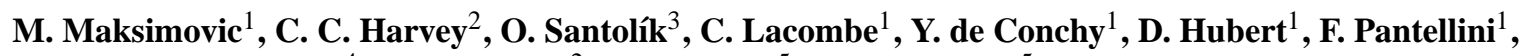 \\ N. Cornilleau-Werhlin ${ }^{4}$, I. Dandouras ${ }^{2}$, E. A. Lucek ${ }^{5}$, and A. Balogh ${ }^{5}$ \\ ${ }^{1}$ DESPA, Observatoire de Paris, France \\ ${ }^{2}$ CESR, Toulouse, France \\ ${ }^{3}$ Department of Physics and Astronomy, University of Iowa, USA \\ ${ }^{4}$ CETP/UVSQ, Vélizy, France \\ ${ }^{5}$ Imperial College, London, UK
}

Received: 17 April 2001 - Revised: 21 June 2001 - Accepted: 23 June 2001

\begin{abstract}
We present observations of "lion roars" obtained in the magnetosheath by the Spectrum Analyser (SA) of the Spatio-Temporal Analysis of Field Fluctuations (STAFF) experiment aboard Cluster. STAFF-SA calculates, in near real time, the complete auto- and cross-spectral matrix derived from three magnetic and two electric components of the electromagnetic field at 27 frequencies in the range of $8 \mathrm{~Hz}$ to $4 \mathrm{kHz}$. This allows the study of the properties of whistler mode waves and more particularly, the properties of "lion roars", which are intense, short-duration, narrowbanded packets of whistler waves. Their presence is favoured by the magnetic field troughs associated with mirror mode structures. During two short periods of well-defined mirror modes, we study the depth $\delta B / B$ of the magnetic troughs, and the direction of propagation of the lion roars. During the first period, close to the magnetopause, deep magnetic troughs pass the satellites. Lion roars are then observed to propagate simultaneously in two directions, roughly parallel and anti-parallel to the magnetic field: this probably indicates that during this period, the satellites were within the successive source regions of lion roars. For the second period, far from the magnetopause, the magnetic troughs are less deep. Lion roars are propagating in only one direction, roughly anti-parallel to the magnetic field, suggesting that the source regions are more distant and predominantly on one side of the satellites.
\end{abstract}

Key words. Magnetospheric physics (magnetosheath; plasma waves and instabilities) Radio science (radiowave propagation)

\section{Introduction}

After a perfect launch and a successful six months of commissioning, the Cluster spacecraft began to execute the Mas-

Correspondence to: M. Maksimovic

(milan.maksimovic@obspm.fr) ter Science Plan on 1 February 2001. We present the first observations made in the magnetosheath with the Spectrum Analyser (SA) of the Spatio-Temporal Analysis of Field Fluctuations (STAFF) experiment. We analyse data obtained on 10 December 2000 , during the commissioning phase of the mission. The STAFF-SA experiment samples the waveform of the three magnetic and two electric components of the electromagnetic field, and then calculates the corresponding $5 \times 5$ Hermitian cross-spectral matrix at 27 frequencies in the range of $8 \mathrm{~Hz}$ to $4 \mathrm{kHz}$.

This frequency range is well-suited for the study of whistler mode waves, which are observed throughout the Earth's magnetosheath between roughly $3 \mathrm{~Hz}$ and $300 \mathrm{~Hz}$ (Smith et al., 1967). The highest occurrence rate of these waves is found in the inner region of the sunward magnetosheath, and the distribution of their intensity at $200 \mathrm{~Hz}$ is observed to peak near the sub-solar magnetopause (Rodriguez, 1985). "Lion roars" (LRs) are intense, short-duration, narrow-band packets of such whistler waves. Whistler waves can be unstable in the magnetosheath since the electron temperature $T_{e \perp}$ perpendicular to the magnetic field is slightly larger than the parallel temperature $T_{e \|}$. LRs are often located in magnetic field troughs associated with density peaks, which are typical of mirror mode structures (Tsurutani et al., 1982). This is due to the fact that for a given ratio $T_{e \perp} / T_{e \|}$, the number of resonant electrons involved in the growth of the whistler instability is larger in the magnetic troughs (Smith and Tsurutani, 1976). Most of the LRs are observed to propagate at small angles $\left(\theta_{k B} \leq 30^{\circ}\right)$ between the directions of the wave vector $\boldsymbol{k}$ and the magnetic field $\boldsymbol{B}$ (Smith and Tsurutani, 1976). Using the high time resolution of the Equator-S magnetometer, Baumjohann et al. (1999) observed that $\theta_{k B}$ was usually smaller than $1.5^{\circ}$, for a sample of nearly monochromatic magnetic waveforms with durations of $\simeq 0.25 \mathrm{~s}$, detected in the deepest part of the magnetic field troughs. Zhang et al. (1998) found that $30 \%$ of the LRs in the magnetosheath were associated with the magnetic troughs of mirror modes. Most of these LRs were propagat- 

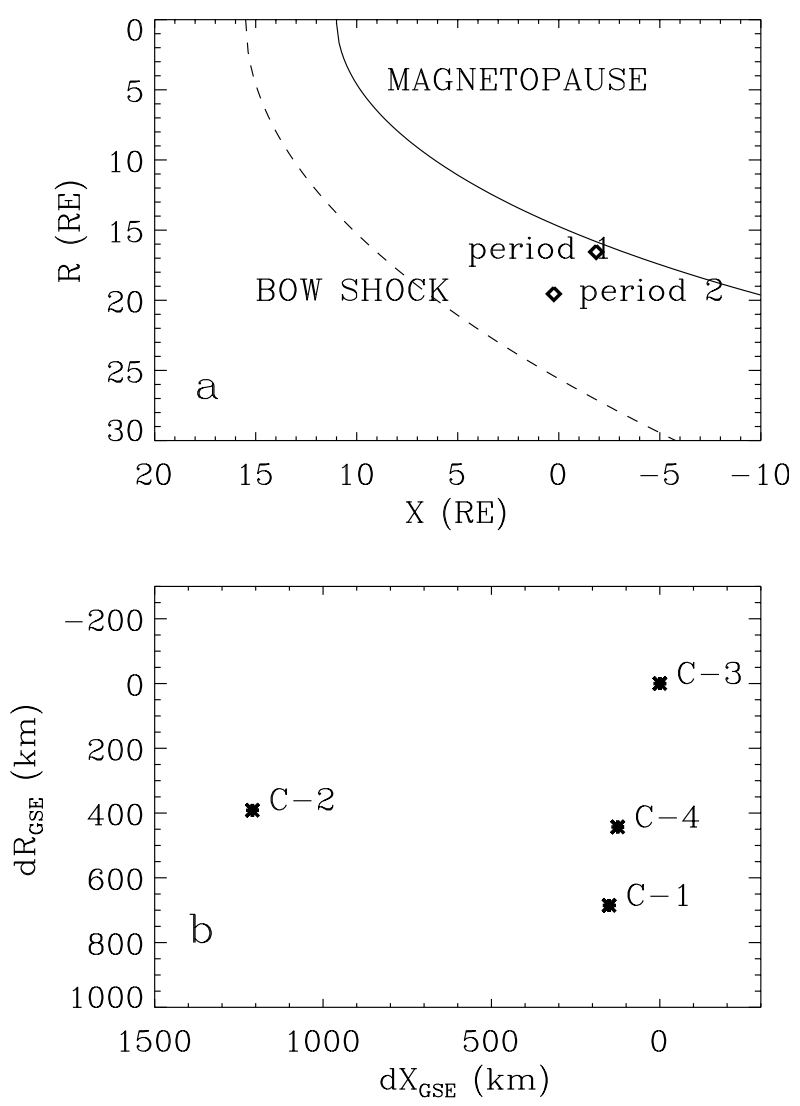

Fig. 1. (a) Geometry during the two periods of observation of lion roars in the dusk side magnetosheath. The coordinates $X$ and $\left.R=\sqrt{(} Y^{2}+Z_{\mathrm{GSE}}^{2}\right)$ are not exactly GSE coordinates, but take into account an aberration of $2.5^{\circ}$ in the ecliptic plane due to the solar wind velocity $V_{\mathrm{sw}} \simeq 650 \mathrm{~km} / \mathrm{s}$ (data from the Wind spacecraft). The magnetopause model used is that of Sibeck et al. (1991) corresponding to the observed solar wind pressure $\simeq 2 \mathrm{nPa}$. (b) Positions of the four spacecraft with respect to Cluster 3 (C-3) during Period 2 in GSE coordinates; $d X_{\mathrm{GSE}}$ is the distance along the GSE $X$-axis and $\left.d R_{\mathrm{GSE}}=\sqrt{(} d Y_{\mathrm{GSE}}^{2}+d Z_{\mathrm{GSE}}^{2}\right) \cdot \mathrm{C}-2$ is more than $1000 \mathrm{~km}$ upstream of the other three spacecraft.

ing in only one direction, which was either parallel or antiparallel to $\boldsymbol{B}$. In a few cases, wave vectors were observed in the two directions, but not at the same time and at the same frequency. In order to determine the direction of the LRs wave vectors without ambiguity of sense, Zhang et al. (1998) used simultaneous observations of both magnetic and electric waveforms.

Assuming that the electromagnetic fluctuations are due to a dominant plane wave, the $5 \times 5$ cross-spectral matrix enables the direction of the wave vector $\boldsymbol{k}$ to be determined with no ambiguity of sense (Santolík et al., 2001). In Sect. 2, we describe briefly the STAFF-SA experiment. In Sect. 3, we present selected STAFF-SA observations of whistler fluctuations and LRs in the magnetosheath. In Sect. 4, we present the method used to determine the polarisation and direction of propagation of these waves, and the results of this analysis. In Sect. 5, we discuss these results and present some
Table 1. The averaged orbital position, magnetic field and plasma convection velocity at the Cluster 3 spacecraft during the two selected periods. All vectors are expressed in GSE coordinates

\begin{tabular}{ccc}
\hline & Period 1 & Period 2 \\
\hline Time & $02: 14: 24$ & $11: 33: 54$ \\
interval & $-02: 16: 12$ & $-11: 38: 24$ \\
\hline$r_{x}$ & -1.23 & 1.07 \\
$r_{y} \quad R_{E}$ & 14.32 & 18.95 \\
$r_{z}$ & 8.41 & 4.66 \\
$|r|$ & 16.65 & 19.54 \\
$B_{x}$ & 14.3 & 0.3 \\
$B_{y} \quad \mathrm{nT}$ & -2.9 & -3.1 \\
$B_{x}$ & -7.3 & 16.0 \\
$|B|$ & 16.3 & 16.30 \\
$V_{x}$ & -362.6 & -358.7 \\
$V_{y} \mathrm{~km} / \mathrm{s}$ & 121.6 & 228.1 \\
$V_{z}$ & 101.4 & 52.0 \\
$|V|$ & 396 & 428 \\
$\theta_{\boldsymbol{V} \boldsymbol{B}}$ & $166.6^{\circ}$ & $89.8^{\circ}$ \\
\hline
\end{tabular}

perspectives.

\section{The STAFF spectrum analyser (SA)}

The STAFF experiment comprises a boom-mounted tri-axial search coil magnetometer and two complementary signal analysis packages: a wave-form receiver, and an onboard digital spectrum analyser (Cornilleau-Wehrlin et al., 1997). In addition to the three magnetic signals, STAFF-SA receives the signals from the four electric probes of the EFW experiment (Gustafsson et al., 1997). These are connected so as to form a pair of orthogonal electric field dipole sensors aligned with the spin-plane magnetic sensors. All five inputs $(3 \times \boldsymbol{B}+$ $2 \times \boldsymbol{E})$ are used to compute in near real time the $5 \times 5$ Hermitian cross-spectral matrix $\mathbf{S}$ at 27 frequencies distributed logarithmically in the frequency range of $8 \mathrm{~Hz}$ to $4 \mathrm{kHz}$. This frequency range is itself divided into three logarithmically distributed frequency sub-bands, each with a maximum frequency eight times the minimum frequency (band A: 8$64 \mathrm{~Hz}$; band B: $64-512 \mathrm{~Hz}$; band C: $512-4096 \mathrm{~Hz}$ ). The pairs of spin-plane magnetic and electric field components are despun aboard the spacecraft. All channels are sampled simultaneously, and the integration time for each channel is the same as the overall instrument time resolution, which can be commanded to values between $125 \mathrm{~ms}$ (except for band A) and $4 \mathrm{~s}$. The five auto-spectral power densities are obtained with a dynamic range of approximately $100 \mathrm{~dB}$ and resolution of $0.38 \mathrm{~dB}$. The 10 complex cross-spectral power estimates are normalised to give the coherence (CornilleauWehrlin et al., 1997). The precision of the phase determination depends upon the magnitude of the coherence: for a 


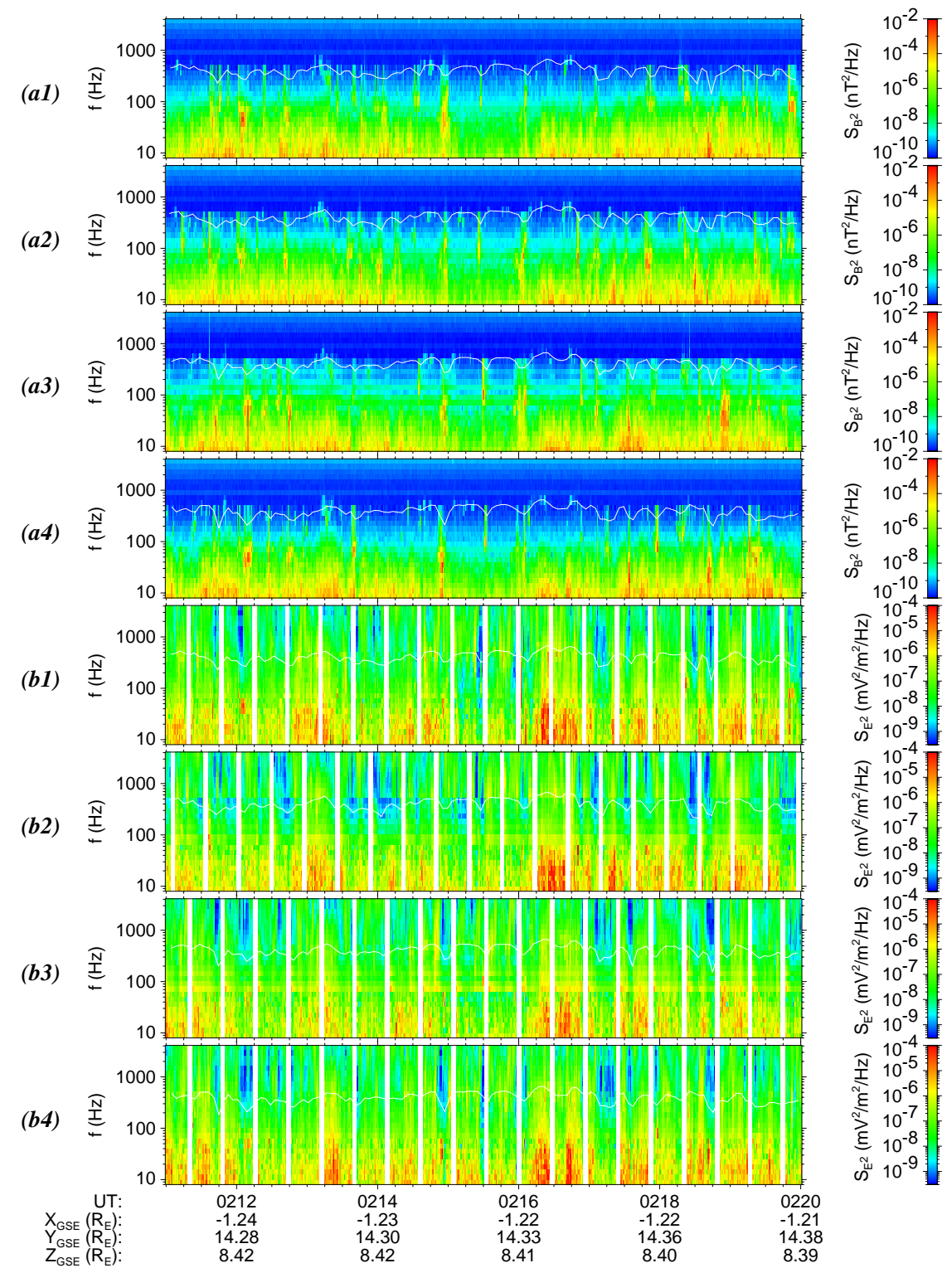

Fig. 2. From 02:10 to 02:20 UT on the 10 Dec. 2000 (Period 1), spectrograms for the four Cluster spacecraft of the magnetic (a1, a2, a3, a4) and electric (b1, b2, b3, b4) auto-spectral power densities. The thin white lines represent the profiles of the electron gyrofrequency $F_{c e}$.

signal with coherence $>50 \%$, it is $\approx 5^{\circ}$ for phases close to $0^{\circ}, 180^{\circ}$, or $\pm 90^{\circ}$, and increases to $\approx 10^{\circ}$ for phases midway between these angles.

In this study, we use data acquired in the normal mode of operation, in which the auto-spectral power densities are determined every second, and the cross-spectral densities every $4 \mathrm{~s}$.

\section{Whistler fluctuations and lion roars in the magne- tosheath}

We present observations obtained by STAFF-SA during two periods of approximately $10 \mathrm{~min}$ duration on 10 December 2000, in the dusk-side magnetosheath. In Fig. 1, the spacecraft positions are shown for the two periods of interest. Typical values of the average DC magnetic field and the pro- ton bulk speed for these two periods, respectively, from the FGM (Balogh et al., 1997) and CIS (Rème et al., 1997) experiments, are summarised in Table 1 . The main difference between these two periods is the angle between the average magnetic field $\boldsymbol{B}$ and the bulk speed $\boldsymbol{V}$. For Period 1, $\boldsymbol{B}$ and $\boldsymbol{V}$ are almost anti-parallel, while for Period 2, they are perpendicular.

In Fig. 2, we display spectrograms for the four Cluster spacecraft showing as functions of frequency and time, the total magnetic $S_{B^{2}}$ and partial electric $S_{E^{2}}$ auto-spectral power densities for Period 1. These total power densities are the sums of the three magnetic and two electric components, i.e. $S_{B^{2}}=S_{B_{x}^{2}}+S_{B_{y}^{2}}+S_{B_{z}^{2}}$ and $S_{E^{2}}=S_{E_{x}^{2}}+S_{E_{y}^{2}}$, the $x$ and $y$ components are in the spacecraft spin plane, and the $z$ component is along the spin axis. In Fig. 3, we display the same quantities for Period 2. In both figures, the elec- 


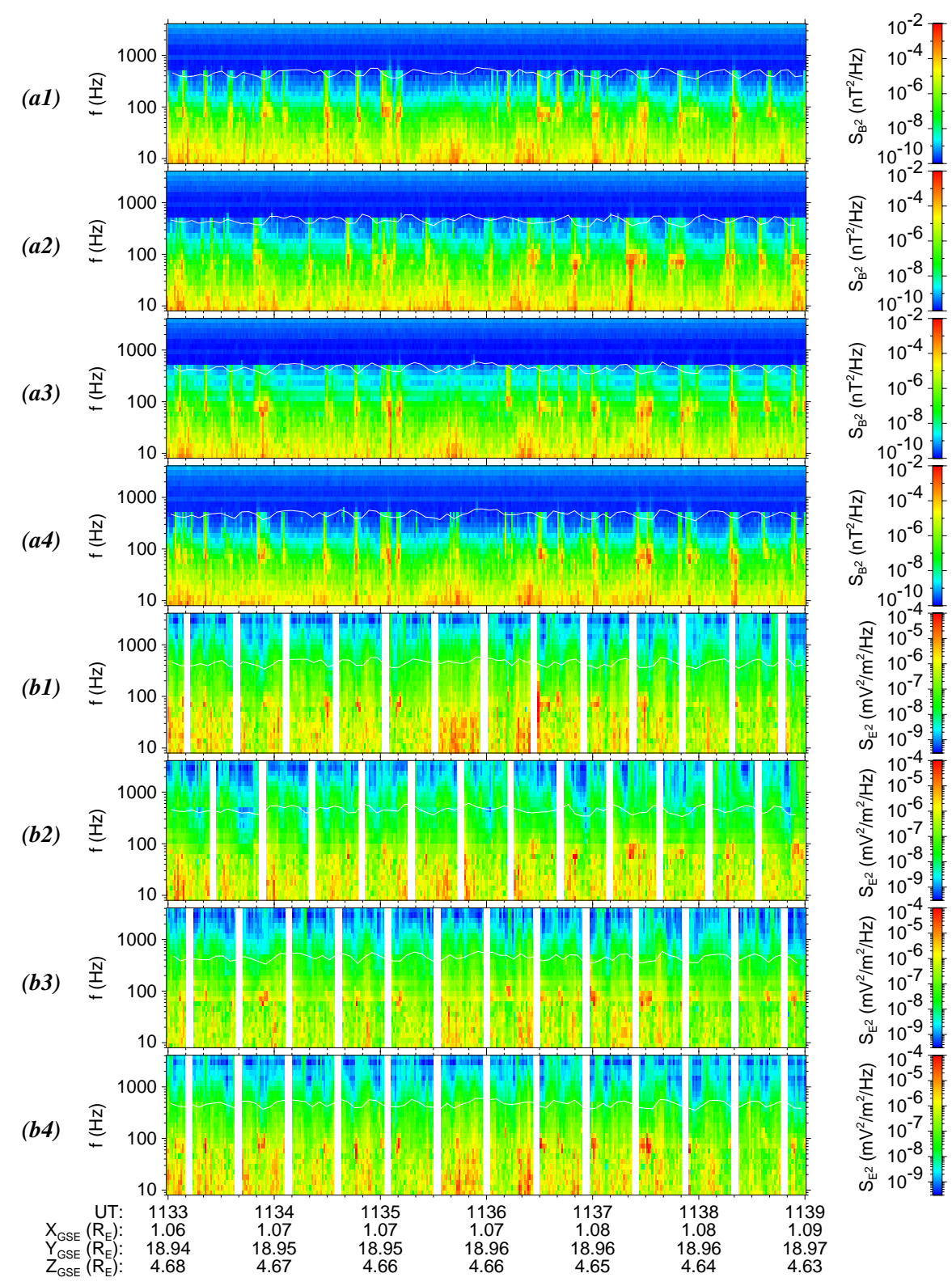

Fig. 3. Same as Fig. 2, from 11:33 to 11:39 UT (Period 2). tron gyro-frequency $F_{\text {ce }}$ is superimposed on the eight spectrograms. $F_{\text {ce }}$ is computed from the spin averaged $(4 \mathrm{~s})$ FGM DC magnetic field. On all the spectrograms, one can clearly distinguish two different types of emission: (i) broadband fluctuations are present almost continuously in the lowest two STAFF-SA frequency bands $(8 \mathrm{~Hz}$ to $512 \mathrm{~Hz}$ ); (ii) superimposed on these fluctuations, more intense and narrowbanded emissions occur sporadically at frequencies between $\approx 40 \mathrm{~Hz}$ and $\approx 200 \mathrm{~Hz}$. Intermittent emissions of this second type are almost always associated with magnetic troughs. This close association suggests that these emissions are examples of intermittent whistler mode lion roars which have been observed for more than 25 years in the magnetosheath. These waves are often associated with the presence of mirror modes which can be the source regions and the wave guides for the LRs in the magnetosheath. The convected mirror mode structures, which are stationary in the plasma frame, are observed around $0.04 \mathrm{~Hz}$ in the satellite frame.

Signals with frequencies around and slightly above the electron gyrofrequency $\left(F_{c e}\right)$ are also visible in Figs. 2 and 3. These signals are generally background rises which are due to the fact that the 8-bit numerisation of the signal by the STAFF-SA receivers does not allow for a dynamic range larger than $\approx 45 \mathrm{~dB}$ in each of the three frequency bands taken separately. When a LR occurs in one band (usually band B) with a signal level $45 \mathrm{db}$ above the noise level, the background noise in increased in that band but not in the others. This causes these signals around and above $F_{c e}$, which then disappear abruptly at the last frequency channel of band $\mathrm{B}$ and do not extend in band $\mathrm{C}$. 
Since the present paper uses data recorded during the commissioning phase of the mission and a spin averaged magnetic field data, we do not have all the information required to completely determine the properties of the mirror modes which we have identified by the regular succession of peaks and troughs of the field modulus. We can, however, state that these fluctuations of the field around $0.04 \mathrm{~Hz}$ are most likely to be mirror mode related: (1) The direction of maximum variance of the magnetic field lies at a small angle to the average field $\boldsymbol{B}_{0}$ (Hubert et al., 1998; Lucek et al., 1999): this is typical of a compressive mode, be it fast, slow or mirror; (2) The minimum variance direction $\boldsymbol{k}_{M}$ is at an angle of more that $80^{\circ}$ from $\boldsymbol{B}_{0}$ (quasi-perpendicular propagation); (3) The Alfvén ratio

$$
R_{A p}=\frac{\delta V^{2} B_{0}^{2}}{\delta B^{2} v_{A}^{2}}
$$

is typical of fluctuations with a dominant mirror mode.

We calculated $R_{A p}$ from the variances $\delta V^{2}$ and $\delta B^{2}$ of the three components of the spin averaged bulk velocity and of the magnetic field. The density is 4.5 to $5 \mathrm{~cm}^{-3}$, the Alfvén speed $v_{A}$ is 160 to $170 \mathrm{~km} / \mathrm{s}$, giving $R_{A p} \simeq 0.17$ to 0.26 for the two selected periods of Table 1 . The proton temperature is $T_{p} \simeq 310^{6} \circ \mathrm{K}$, so that the local plasma $\beta$ is about 2 . As shown, for instance, by Denton et al. (1998), when $\beta>1$, the only mode which propagates quasi-perpendicularly with $R_{A p}<1$ is the mirror mode (Czaykowska et al., 1998).

The characteristic dimensions of these mirror structures can be determined during the two selected periods. During the first interval (see Table 1), the plasma velocity $\boldsymbol{V}$ makes a small angle with $\boldsymbol{B}_{0}$. The time intervals between successive magnetic troughs of $B_{0}$ thus give a scale between 6700 and $14000 \mathrm{~km}$ along $\boldsymbol{B}_{0}$. For the second period, the plasma velocity $\boldsymbol{V}$ makes an angle of less than $17^{\circ}$ with the direction $\boldsymbol{j}=\boldsymbol{k}_{\boldsymbol{M}} \times \boldsymbol{B}_{\mathbf{0}}, \boldsymbol{k}_{\boldsymbol{M}}$ being the minimum variance direction. We then find a length scale between 7000 and $9000 \mathrm{~km}$ along $j$ between successive minima of $B_{0}$. The picture we obtain from this analysis is that of mirror structures draping the magnetopause surface, with the major axis along $\boldsymbol{B}_{0}$, the intermediate axis along a direction orthogonal to $\boldsymbol{B}_{0}$ and $\boldsymbol{k}_{\boldsymbol{M}}$, and the probable minor axis along $\boldsymbol{k}_{\boldsymbol{M}}$ (Fazakerley et al., 1994; Hubert et al., 1998).

In Fig. 4a, we plot for Period 1 and for each of the four spacecraft, the average spectra of $S_{B^{2}}$ as a function of $F / F_{c e}$, where $F_{c e}$ is obtained by interpolation between consecutive spin-averaged magnetic field values. We have selected only the data contained in the lowest two frequency bands of STAFF-SA, in the range of $8 \mathrm{~Hz}$ to $512 \mathrm{~Hz}$. The diamonds connected with a solid line represent the sensitivity level of STAFF search-coil. This background level is well below the signal we studied. The four spacecraft show very similar spectra with a bump at $F / F_{c e} \approx 0.2$. In order to select the intermittent LR events for this period, we plot in Fig. $4 \mathrm{~b}$ the probability distributions of $\log _{10}\left(S_{B^{2}}\right)$ for all the frequency channels corresponding to the spectral bump of Fig. 4a. More precisely, we select all the frequency channels satisfying the condition $0.1 \leq F / F_{c e} \leq 0.3$, as indicated by the two dashed
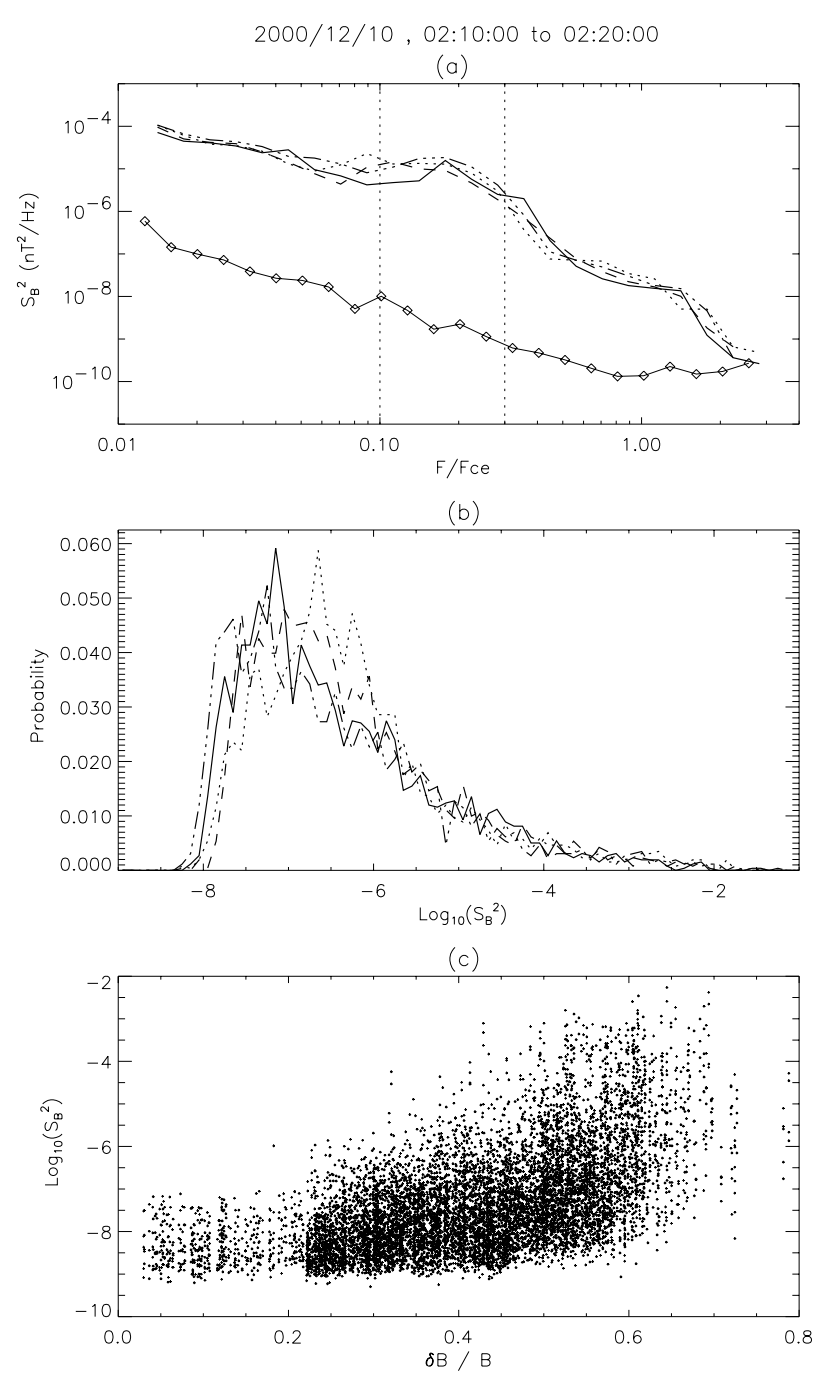

Fig. 4. For Period 1: (a) average spectra of the magnetic fluctuations on C1 (solid line), C2 (dashed line), C3 (dotted line) and C4 (dash-dot-dot-dot line), as functions of the normalised frequency $F / F_{c e}$. The vertical dotted lines select the $F / F_{c e}$ subsets used in the bottom panels, $0.1<F / F_{c e}<0.3$. The diamonds indicate the sensivity of the search coils. (b) histograms of the spectral power $S_{B^{2}}$, in the $F / F_{c e}$ subset, for the four spacecraft. (c) for the $F / F_{c e}$ subset and the four spacecraft, scatter plot of $S_{B^{2}}$ as a function of the relative depth $\delta B / B$ of the magnetic field with respect to the maximum field magnitude $B$ (see Sect. 3 ).

vertical lines in Fig. 4a. We will call these subsets of data the $F / F_{\text {ce }}$ subsets. The probability distributions of $\log _{10}\left(S_{B^{2}}\right)$ are very similar for the $F / F_{\text {ce }}$ subsets obtained from each of the four spacecraft. The distributions are log-normal with a high-power tail characteristic of intermittent events. With the help of these distributions, we recover the intermittent LRs from the background fluctuations by simply selecting the events with $S_{B^{2}} \geq 10^{-5} \mathrm{nT}^{2} / \mathrm{Hz}$. Figures $5 \mathrm{a}$ and $5 \mathrm{~b}$ represent the same analysis for Period 2. In this case, one observes that for the average $S_{B^{2}}$ spectra, the peak at $F / F_{c e} \approx 0.2$ is more prominent. Furthermore, the $\log _{10}\left(S_{B^{2}}\right)$ probability distributions of the $F / F_{c e}$ subsets exhibit more significant 

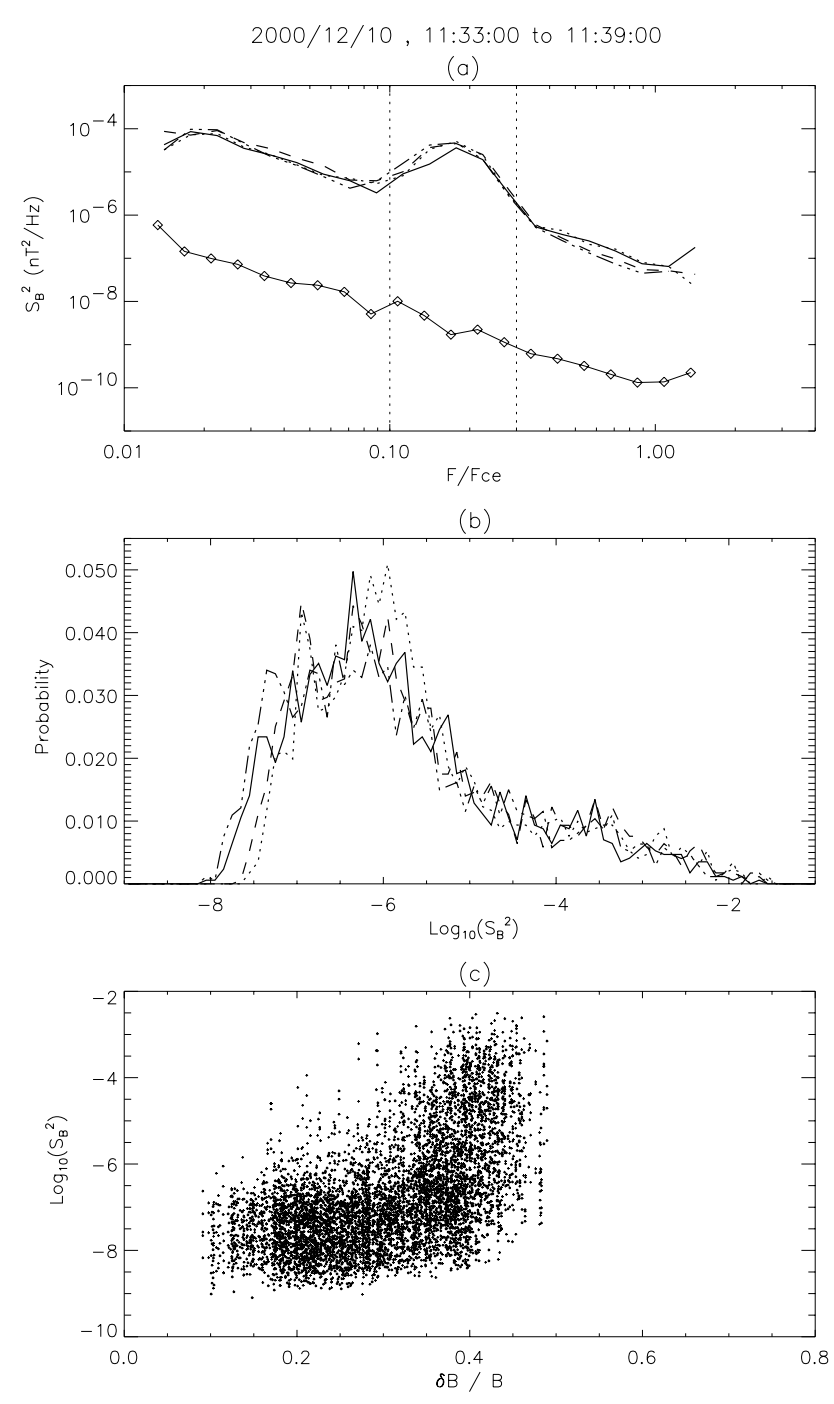

Fig. 5. Same as Fig. 4, for Period 2.

high-power tails.

Figures $4 \mathrm{c}$ and $5 \mathrm{c}$ show the scatter plot of $\log _{10}\left(S_{B^{2}}\right)$ as a function of $\delta B / B$, for the ensemble of the $F / F_{c e}$ subsets from all four spacecraft. For each individual $F / F_{c e}$ event $i$, $\delta B / B$ is defined as $(\delta B / B)_{i}=\left(B^{\star}-B_{i}\right) / B^{\star}$, where $B_{i}$ is the value of modulus of the spin averaged magnetic field (of the appropriate spacecraft) interpolated for the time of the event $i$, and $B^{\star}=<B>+3 \sigma_{B}$, where $<B>$ is the mean magnetic field intensity during Period 1 (or 2) and $\sigma_{B}$ is the standard deviation of its distribution. $B^{\star}$ is thus a local measure of the maximum magnetic field strength, and $\delta B$ is the local depth of the magnetic troughs. We note that the range of $\delta B / B$ is rather different for the two periods. To see this difference better, we plot in Fig. 6 the probability distributions of $\delta B / B$ for the two periods. The solid lines represent this distribution for the LR events, extracted from the $F / F_{c e}$ subsets by selecting the events with $S_{B^{2}} \geq 10^{-5} \mathrm{nT}^{2} / \mathrm{Hz}$, and the dashed lines represent the distributions of $\delta B / B$ for the non-LR events with $S_{B^{2}}<10^{-5} \mathrm{nT}^{2} / \mathrm{Hz}$. The $\delta B / B$ distri-
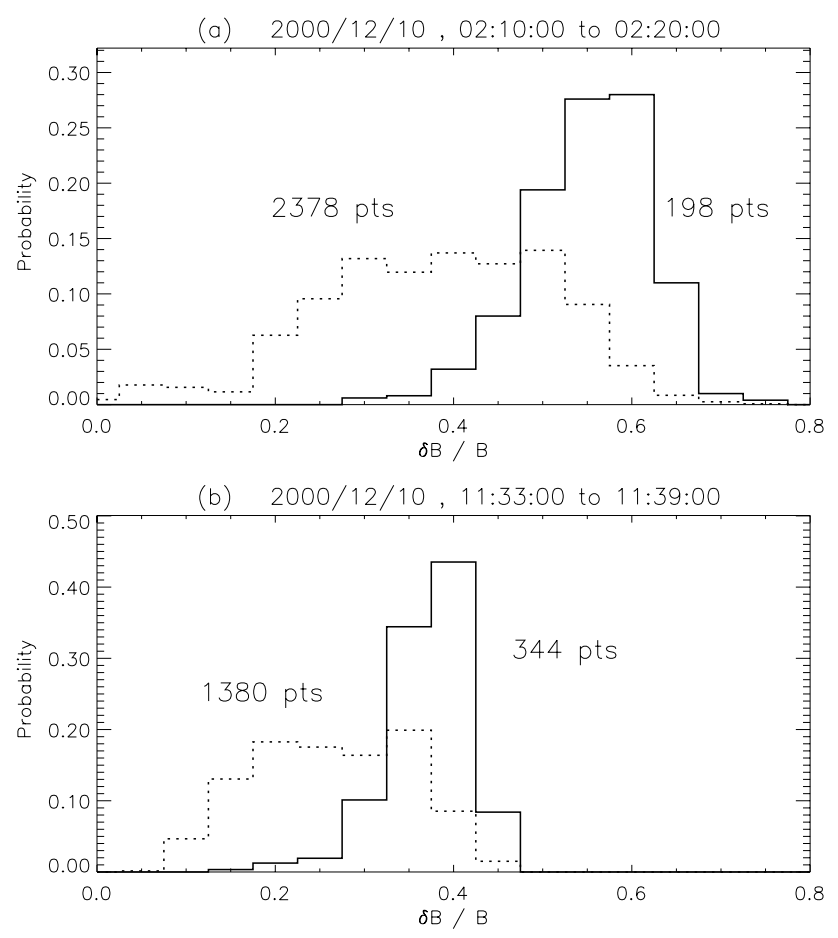

Fig. 6. For the $F / F_{c e}$ subsets, (a) histogram of the relative depth $\delta B / B$ of the magnetic field observed at the times of the lion roars (solid line), and at the times of the other whistlers at the same frequencies, for Period 1; (b) the same histograms for Period 2.

bution extends up to $\approx 0.7$ for Period 1 and up to $\approx 0.5$ for Period 2. This difference is most likely due to the fact that during Period 1, the spacecraft were closer to the magnetopause than during Period 2, and the convected mirror mode fluctuations were more developed. For the LR events, the distribution of $\delta B / B$ lies between $\approx 0.3$ and $\approx 0.7$ for Period 1 , and between $\approx 0.2$ and $\approx 0.5$ for Period 2 . From this analysis, we conclude that LRs do not seem to be triggered by a fixed $\delta B / B$ amplitude. However, the LRs occupy for each of the two periods a comparable portion of the overall $\delta B / B$ distributions: they start at roughly the mean value of these distributions. This analysis is still preliminary, and in a future study, we plan to use the highest resolution data from both the magnetometer and STAFF to test the model of Lee et al. (1987) for the growth of LRs in magnetic mirror mode structures, and the model of Treumann et al. (2000) for the trapping of LRs in the associated density peaks.

\section{Polarisation and direction of propagation of whistler waves}

We now present the results of the analysis of the wave polarisation and propagation properties of the data $F / F_{c e}$ subsets. This analysis is performed using several different methods coded in the computer program PRASSADCO (Santolík, 2000), which has been especially developed for the analysis of the STAFF-SA data. 

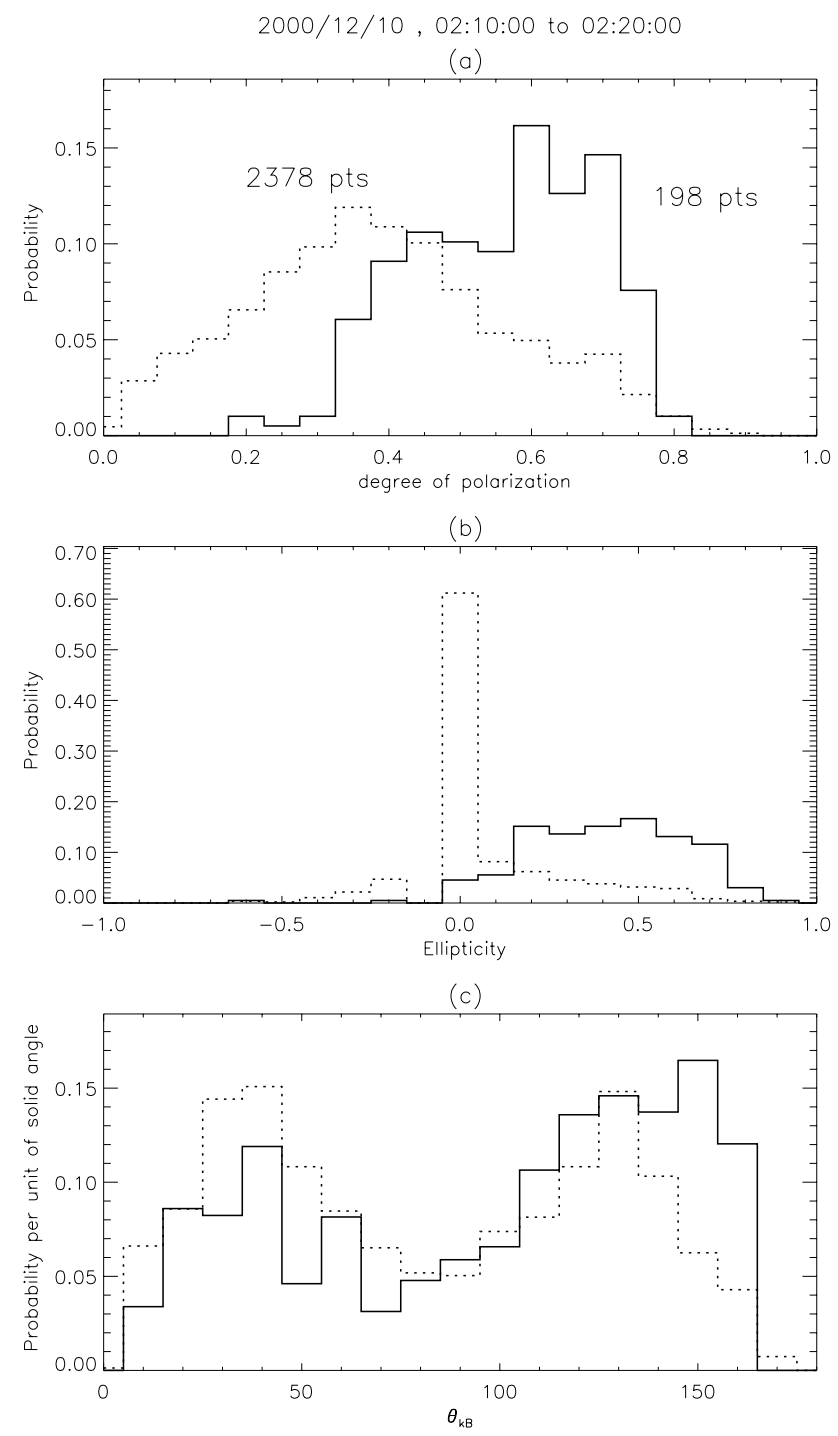

Fig. 7. Histograms for Period 1 and for the $F / F_{c e}$ subset of: (a) the degree $P$ of polarisation (Eq. 1), (b) the ellipticity ( +1 for a circular right-hand polarisation), (c) the angle between the wave vector and $\boldsymbol{B}_{0}$, for the lion roars (solid line) and for the other whistlers (dotted line).

\subsection{Analysis methods}

To measure the 3D coherence of the magnetic field components, we use the degree of polarisation defined by Samson and Olson (1980),

$P^{2}=\frac{3}{2} \operatorname{tr} \mathbf{S}^{2} /(\operatorname{tr} \mathbf{S})^{2}-\frac{1}{2}$,

where $\operatorname{tr} \mathbf{S}$ is the trace of the spectral matrix $\mathbf{S}$ and $\operatorname{tr} \mathbf{S}^{2}$ is the trace of $\mathbf{S} \times \mathbf{S}$. The value of $P$ has the following significance: $P$ is zero if the spectral power is isotropic and completely unpolarised, and $P=1$ if the three field components are fully coherent and the wave field is fully polarised.

Successive spectral matrices are despun aboard the spacecraft and are obtained in a Cartesian coordinate system with
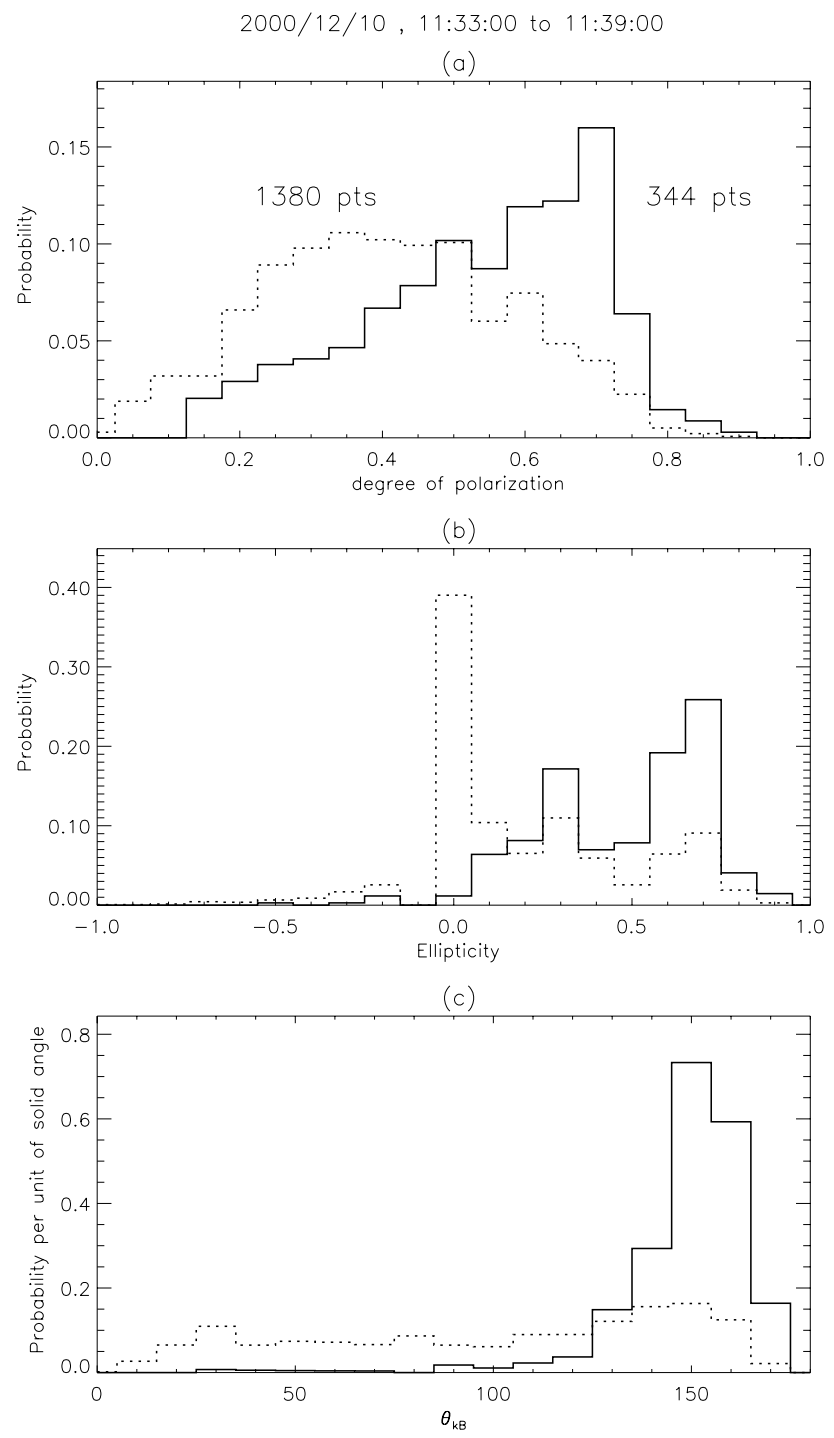

Fig. 8. Same as Fig. 7, for Period 2.

the $z$-axis along the satellite spin axis and the $x$-axis orthogonal to the $z$-axis, in the plane of the $z$-axis and the direction of the Sun. Since we can suppose that the plasma is locally symmetric about the magnetic field direction, we transform the matrices to the coordinate system defined by the local DC magnetic field. In this frame, the $z$-axis is parallel to the magnetic field and the $x$-axis lies in the plane defined by the magnetic field and the direction of the Sun. The transformed matrices are used to determine the polarisation of the magnetic fluctuations with respect to the DC magnetic field. This polarisation is defined by the phase shift between the $x$ and $y$ components, i.e. by the sign of the imaginary part of their cross-spectrum. By convention, we choose a positive sign for the right-hand polarisation, corresponding to the sense of gyration of the electron. In our results, this sign is combined with the ratio of the axes of the polarisation ellipse (ellipticity) obtained by the method of Samson and Olson (1980). 

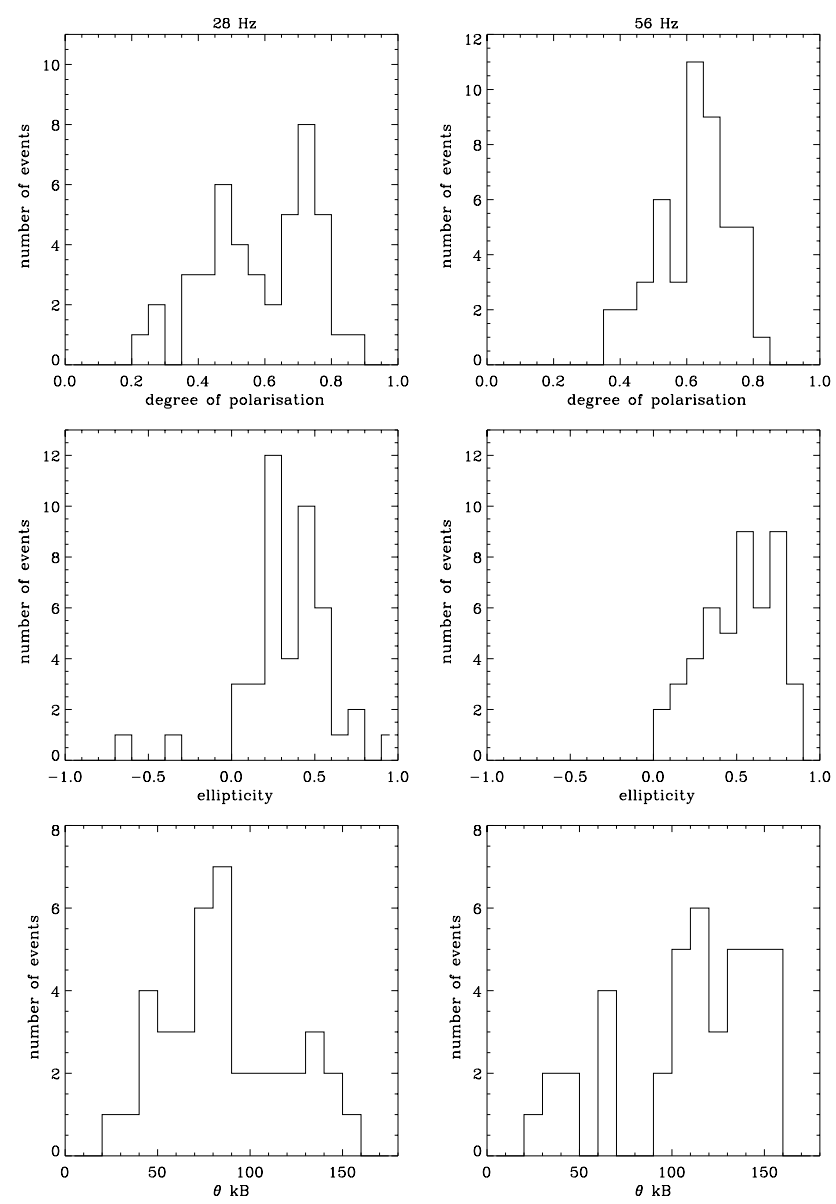

Fig. 9. For Period 1, histograms of the LR properties $\left(S_{B^{2}} \geq\right.$ $10^{-5} \mathrm{nT}^{2} / \mathrm{Hz}$ ) for two specific frequencies of the STAFF-SA receiver: $28 \mathrm{~Hz}$ (left panels) and $56 \mathrm{~Hz}$ (right panels).

For a single monochromatic linear plane wave, Faraday's law can be written as

$n(\boldsymbol{\kappa} \times \boldsymbol{E})=c \boldsymbol{B}$

where $\boldsymbol{E}$ and $\boldsymbol{B}$ are the complex amplitudes of the electric and magnetic field fluctuations, $n$ is the index of refraction $n=k c / \omega, k$ is the wavenumber, $c$ is the speed of light, $\omega$ is the angular frequency, and $\boldsymbol{\kappa} \equiv \boldsymbol{k} / k$. From Eq. (2), it follows that the wave vector is perpendicular to the wave magnetic field, i.e. that $\boldsymbol{k} \cdot \boldsymbol{B}=0$. This general property is often used to estimate the wave vector direction (Means, 1972).

Note that if the polarisation is circular or elliptic, two mutually anti-parallel directions satisfy the condition of perpendicularity. The determination of the wave vector direction is thus ambiguous. The complete wave vector direction without this ambiguity can be determined using both the magnetic field and the electric field (Lefeuvre et al., 1986-7; Santolík and Parrot, 1999). Having the magnetic field vector data and the two electric components, we use Eq. (2) to calculate the third electric component, as described by Santolík et al. (2001). The information on all six field components can be inserted back into Eq. (2), and the complete wave vector di- rection can be evaluated. We use a method based on the singular value decomposition (SVD) algorithm and we obtain a wave vector direction $\theta_{k B}$ which has the optimum agreement with all six field components (Santolík et al., 2000). $\theta_{k B}$ varies from 0 ( $\boldsymbol{k}$ parallel to the DC magnetic field $\left.\boldsymbol{B}_{0}\right)$ to $180^{\circ}$ ( $\boldsymbol{k}$ anti-parallel to $\boldsymbol{B}_{0}$ ).

4.2 A statistical analysis of the whistlers and lion roars polarisation and propagation properties

The results of our polarisation and propagation analysis for Periods 1 and 2 are plotted, respectively, in Figs. 7 and 8; the three panels show the probability distributions of the degree of polarisation (panel a) and the ellipticity (panel b), and of the normalised (per unit solid angle) probability of the angle $\theta_{k B}$ between $\boldsymbol{k}$ and $\boldsymbol{B}_{0}$ (i.e. the probability divided by $\left.\sin \theta_{k B}\right)$. Only data from the $F / F_{c e}$ subsets has been used. The solid lines represent the probability distributions for LR events, which are selected from the $F / F_{c e}$ subsets by the criterion $S_{B^{2}} \geq 10^{-5} \mathrm{nT}^{2} / \mathrm{Hz}$ and the dashed lines represent the probability distributions of events not satisfyng this condition, the non-LR events.

During both periods, compared to the non-LR events, the LR events have a larger degree of polarisation. Their polarisation is more circular and more right-handed. But the distributions of $\theta_{k B}$ are different for the two periods. Fig. 7 shows that during Period 1, the distribution of $\boldsymbol{k}$ has two peaks, the larger one roughly anti-parallel to $\boldsymbol{B}_{0}$, the other roughly parallel to $\boldsymbol{B}_{0}$. During this period, the plasma velocity (Table 1) was nearly anti-parallel to $\boldsymbol{B}_{0}$, so that LRs are directed towards both the downstream and upstream regions. We must, therefore, study possible Doppler effects. For this purpose, in Fig. 9, we display the histograms of the properties of LRs $\left(S_{B^{2}} \geq 10^{-5} \mathrm{nT}^{2} / \mathrm{Hz}\right)$ for two specific frequencies of the STAFF-SA receiver, $28 \mathrm{~Hz}$ and $56 \mathrm{~Hz}$. Since these properties are statistically very similar on all four satellites, we include data from all spacecraft in Fig. 9. At these two frequencies, the degree of polarisation and the ellipticity are quite similar; but the histograms of $\theta_{k B}$ are rather different. At $28 \mathrm{~Hz}$, there are $25 \mathrm{LRs}$ with $\theta_{k B}<90^{\circ}$ (i.e. propagating upstream) and only 14 LRs with $\theta_{k B}>90^{\circ}$ (propagating downstream). Conversely, at $56 \mathrm{~Hz}$, there are only 9 LRs upstream, and 31 downstream. This is simply due to the Doppler shift: downstream waves are seen at higher frequencies, and upstream waves are seen at lower frequencies. For instance, for a given LR on Cluster 3 (Samba) at 02:15 UT, $\theta_{k B}$ is $40^{\circ}$ and $32^{\circ}$ at low frequencies $(35$ and $44 \mathrm{~Hz}$, respectively); and $\theta_{k B}$ is $129^{\circ}$ and $167^{\circ}$ at higher frequencies (56 and $70 \mathrm{~Hz}$, respectively). Therefore, waves with $\boldsymbol{k}$ roughly parallel and anti-parallel to $\boldsymbol{B}_{0}$ are observed simultaneously at different frequencies. A Doppler shift of 10 to $35 \mathrm{~Hz}$ between downstream and upstream propagating waves corresponds to wavelengths of 20 to $80 \mathrm{~km}$, typical of unstable whistlers, and much smaller than the satellite separations. As noted by Zhang et al. (1998), LRs can be observed simultaneously in opposite directions if the satellites are within the source region. This is probably what occurs during Period 1. 
During Period 2 (Fig. 8), $\boldsymbol{k}$ is primarily anti-parallel to $\boldsymbol{B}_{0}$ for the LR events, while $\theta_{k B}$ is almost uniformly distributed over all possible values for the non-LR events. As the $\boldsymbol{B}_{0}$ field direction is close to the $+Z_{G S E}$ direction, the LRs propagate primarily towards lower latitudes. The plasma velocity is nearly perpendicular to $\boldsymbol{B}_{0}$ (Table 1), so that the Doppler effect is negligible. As the LRs propagate in one direction, successive sources of the LRs were probably on the same side of the satellites during this period of $6 \mathrm{~min}$.

Among the LRs observed in mirror mode troughs, Zhang et al. (1998) found that the wave vectors were generally in one direction, parallel or anti-parallel to $\boldsymbol{B}_{0}$; in a few magnetic troughs, LRs were found in both directions, but at slightly different times and at different frequencies. Our results are consistent with these observations. However, we are unable to explain why the magnetic troughs of Period 1 are source regions, providing LRs in two directions, while the magnetic troughs of Period 2 are not source regions and remain on the same side of the source regions. According to Baumjohann et al. (1999), the LRs are primarily found at the very minima of the magnetic troughs. This suggests that the four spacecraft have crossed the very minima of the B field during Period 1 (down to $5 \mathrm{nT}$ ), but not during Period 2 (down to $10 \mathrm{nT}$ ). However, there is no reason for this ad hoc suggestion, since the four spacecraft record nearly the same magnetic profiles during Period 2, despite the separations which are not negligible with respect to the scales of the mirror structures.

Let us finally note that we find LRs propagating at angles of $30^{\circ}$ or $50^{\circ}$ from $\boldsymbol{B}_{0}$, as Lin et al. (1994) did in the Jovian magnetosheath. Such whistlers should not be observed as they usually experience a strong linear Landau damping in a bi-Maxwellian plasma. Smith and Tsurutani (1976) and Baumjohann et al. (1999) found LRs at smaller angles from $\boldsymbol{B}_{0}$, in the Earth's magnetosheath. This difference may have several explanations that we discuss in the next section.

\section{Discussion and perspectives}

We have considered two periods when whistlers and LRs are observed, together with mirror modes, in the dusk side magnetosheath. At frequencies $F>0.1 F_{c e}$, lion roars can be separated from other whistlers because they are more intense and more intermittent. Near the magnetopause (Period 1), LRs propagate at angles around $150^{\circ}$ and $40^{\circ}$ from the $\boldsymbol{B}_{0}$ field, i.e. with $\boldsymbol{k}$ vectors roughly parallel and anti-parallel to $\boldsymbol{B}_{0}$. In the inner magnetosheath (Period 2), where the mirror modes are less intense, we find $\boldsymbol{k}$ at an angle around 150$160^{\circ}$ to $\boldsymbol{B}_{0}$ and directed towards low-latitudes. We do not find wave vectors at very small angles to $\boldsymbol{B}_{0}$ or $-\boldsymbol{B}_{0}$, as did Baumjohann et al. (1999). This difference could be due to the fact that our assumption of a dominant single monochromatic plane wave is not always fulfilled. For example: (i) the cross-spectral elements of the spectral matrix are determined once every $4 \mathrm{~s}$ in normal mode, which is a very low time resolution compared to the waveform analysis of LRs per- formed by Baumjohann et al. (1999); (ii) the STAFF-SA frequency bandwidth is relatively large and proportional to the frequency, which may lead to mixing of several monochromatic plane wave; (iii) to reduce the telemetry load, the digitisation of the cross-spectral matrix elements is adequate to determine the relative phase between two fully coherent input signals with a precision which is at best $5^{\circ}$; however, it seems unlikely that this uncertainty propagates systematically into the determination of $\boldsymbol{k}$.

We plan to analyse the $B$ and $E$ waveforms when STAFF is in its burst mode of operation. It is then possible to recover the waveform for analysis (in the laboratory) at frequencies up to the Nyquist frequency, $\sim 50 \mathrm{~Hz}$, and use a narrower band for short coherent wave packets such as LRs. However, such an analysis is less general because waveforms are selected with variable criteria of coherency, bandwidth and time duration. The advantage of STAFF-SA in normal mode is that it yields the wave vector direction continuously, in LRs as well as in the other whistlers, and in the low frequency whistlers below $0.1 F_{c e}$ (see Figs. 2 and 3). We could also test, by using the four spacecraft with STAFF-SA in its burst mode of data capture (auto-spectra every $0.125 \mathrm{~ms}$ and crossspectra every second), whether the LRs can escape from their mirror magnetic bottles, or if their generation and propagation are purely local. The interesting properties of the LRs outside of the mirror mode regions, described by Zhang et al. (1998), will also be analysed.

Acknowledgements. Topical Editor M. Lester thanks Y. Zhang and another referee for their help in evaluating this paper.

\section{References}

Balogh, A., Dunlop, M. W., Cowley, S. W. H., et al.: The Cluster Magnetic Field Investigation, Space Sci. Rev., 79, 65-92, 1997.

Baumjohann, W., Treumann, R. A., Georgescu, E., Haerendel, G., Fornacon, K.-H., and Auster, U.: Waveform and packet structure of lion roars, Ann. Geophysicae, 17, 1528-1534, 1999.

Cornilleau-Wehrlin, N., Chauveau, P., Louis, S., et al.: The Cluster spatio-temporal analysis of field fluctuations (STAFF) experiment, Space Sci. Rev., 79, 107-136, 1997.

Czaykowska, A., Bauer, T. M., Treumann, R. A., and Baumjohann, W.: Mirror waves downstream of the quasi-perpendicular bow shock, J. Geophys. Res., 103, 4747-4753, 1998.

Denton, R. E., Lessard, M. R., LaBelle, J. W., and Gary, S. P.: Identification of low-frequency magnetosheath waves, J. Geophys. Res., 103, 23 661-23 676, 1998.

Fazakerley, A. N. and Southwood, D. J.: Mirror instability in the magnetosheath, Adv. Space Res., 14(7), 65, 1994.

Gustafsson, G., Boström, R., Holback, B., et al.: The Electric Field and Wave Experiment for the Mission, Space Sci. Rev., 79, 137156, 1997.

Hubert, D., Lacombe, C., Harvey, C. C., Moncuquet, M., Russell, C. T., and Thomsen, M. F.: Nature, properties and origin of low-frequency waves from an oblique shock to the inner magnetosheath, J. Geophys. Res., 103, 26 783-26 798, 1998.

Lee, L. C., Wu, C. S., and Price, C. P.: On the generation of Magnetosheath Lion Roars, J. Geophys. Res., 92, 2343-2348, 1987. 
Lefeuvre, F., Marouan, Y., Parrot, M., and Rauch, J. L.: Rapid determination of the sense of polarization and propagation for random electromagnetic wave fields. Application to GEOS1 and AUREOL3 data, Ann. Geophysicae, 4, 457-468, 1986. (Correction, Ann. Geophysicae, 5, 251, 1987.)

Lin, N., Kellogg, P. J., Thiessen, J. P., Lengyel-Frey, D., Tsurutani, B. T., and Phillips, J. L.: Whistler mode waves in the Jovian magnetosheath, J. Geophys. Res., 99, 23 527-23 539, 1994.

Lucek, E. A., Dunlop, M. W., Balogh, A., Cargill, P., Baumjohann, W., Georgescu, E., Haerendel, G., and Fornacon, K.-H.: Identification of magnetosheath mirror modes in Equator-S magnetic field data, Ann. Geophysicae, 17, 1560-1573, 1999.

Means, J. D.: Use of the three-dimensional covariance matrix in analyzing the polarization properties of plane waves, J. Geophys. Res., 77, 5551-5559, 1972.

Rodriguez, P.: Magnetosheath whistler turbulence, J. Geophys. Res., 90, 6337-6342, 1985.

Rème, H., Bosqued, J. M., Sauvaud, J. A., et al.: The Cluster Ion Spectrometry (CIS) Experiment, Space Sci. Rev., 79, 303-350, 1997.

Samson, J. C. and Olson, J. V.: Some comments on the descriptions of the polarisation states of waves, Geophys. J. R. Astron. Soc., 61, 115-129, 1980.

Santolík, O. and Parrot, M.: Case studies on wave propagation and polarization of ELF emissions observed by Freja around the local proton gyro-frequency, J. Geophys. Res., 104, 2459-2476, 1999.

Santolík, O.: Propagation Analysis of Staff-SA Data with Co- herency Tests, LPCE/NTS/073.B, Lab. Phys. Chimie Environ./CNRS, Orleans, France, 2000.

Santolík, O., Parrot, M., and Lefeuvre, F.: SVD methods for wave propagation analysis, submitted to Radio Sci., 2000.

Santolík, O., Lefeuvre, F., Parrot, M., and Rauch, J. L.: Complete wave-vector directions of electromagnetic emissions: Application to INTERBALL-2 measurements in the nightside auroral zone, J. Geophys. Res., (in press) 2001.

Sibeck, D. G., Lopez, R. E., and Roelof, E. C.: Solar wind control of the magnetopause shape, location and motion, J. Geophys. Res., 96, 5489-5495, 1991.

Smith, E. J. and Tsurutani, B. T.: Magnetosheath lion roars, J. Geophys. Res., 81, 2261-2266, 1976.

Smith, E. J., Holzer, R. E., McLeod, M. G., and Russell, C. T.: Magnetic noise in the magnetosheath in the frequency range 3300 Hz, J. Geophys. Res., 72, 4803-4813, 1967.

Treumann, R. A., Georgescu, E., and Baumjohann, W.: Lion Roar trapping in mirror modes, Geophys. Res. Lett., 27, 1843-1846, 2000.

Tsurutani, B. T., Smith, E. J., Anderson, R. R., Ogilvie, K. W., Scudder, J. D., Baker, D. N., and Bame, S. J.: Lion Roars and nonoscillatory drift mirror waves in the magnetosheath, J. Geophys. Res., 87, 6060-6072, 1982.

Zhang, Y., Matsumoto, H., and Kojima, H.: Lion roars in the magnetosheath: the Geotail observations, J. Geophys. Res., 103, 4615-4626, 1998. 